\title{
Long non-coding RNA GAS5 inhibits ovarian cancer cell proliferation via the control of microRNA-21 and SPRY2 expression
}

\author{
NANA MA, SHAORU LI, QUANHUA ZHANG, HONGMEI WANG, HAIXIA QIN and SHIJIN WANG \\ Department of Gynecology and Obstetrics, The First Affiliated Hospital of Xinxiang Medical University, \\ Weihui, Henan 453100, P.R. China
}

Received August 5, 2017; Accepted January 26, 2018

DOI: $10.3892 /$ etm.2018.6188

\begin{abstract}
In recent decades, numerous long non-coding (lnc) RNAs, including growth arrest-specific transcript 5 (GAS5), have been demonstrated to exert promoting or suppressive effects in human cancers. Decreased expression of the IncRNA GAS5 was reported to promote cell proliferation, migration and invasion and indicate poor prognosis in ovarian cancer. However, the exact underlying molecular mechanism through which GAS5 is involved in ovarian cancer growth remains unknown. The present study aimed to investigate the regulatory mechanism of GAS5 in ovarian cancer cell proliferation. Quantitative polymerase chain reaction and western blot analysis were used to examine RNA and protein expression, respectively. An MTT assay was used to examine cell proliferation. A luciferase reporter gene assay was conducted to verify the targeting relationship. It was identified that the expression levels of GAS5 and Sprouty homolog 2 (SPRY2) were significantly downregulated, while the expression level of microRNA (miR)-21 was significantly upregulated in ovarian cancer tissues and cell lines compared with adjacent non-tumor tissues and normal ovarian epithelial cells, respectively. Downregulation of GAS5 was significantly associated with advanced clinical stage. Luciferase assay data indicated that miR-21 was a direct target of GAS5 and that SPRY2 was a target gene of miR-21 in ovarian cancer-derived A2780 cells. GAS5 overexpression significantly inhibited the proliferation of ovarian cancer cells, which was accompanied by the downregulation of miR-21 and the upregulation of SPRY2. The overexpression of miR-21 caused a significant decrease in A2780 cell proliferation, which was accompanied by reduced
\end{abstract}

Correspondence to: Professor Shijin Wang, Department of Gynecology and Obstetrics, The First Affiliated Hospital of Xinxiang Medical University, 88 Jiankang Road, Weihui, Henan 453100, P.R. China

E-mail: xmuwangshijin@163.com

Key words: ovarian cancer, long non-coding RNA, microRNA, Sprouty homolog 2, proliferation
SPRY2 expression. Furthermore, miR-21 overexpression attenuated the suppressive effects of GAS5 on A2780 cell proliferation and rescued the promoting effects of GAS5 on SPRY2 expression. In addition, the knockdown of SPRY2 also rescued the suppressive effects of GAS5 on the proliferation of A2780 cells. In summary, our study demonstrates that GAS5 exerts a suppressive effect on the proliferation of ovarian cancer cells, at least in part via the inhibition of miR-21 expression and subsequent increased SPRY2 expression. These findings suggest that the GAS5/miR-21/SPRY2 signaling pathway may be a potential therapeutic target in ovarian cancer.

\section{Introduction}

Ovarian cancer is one of the most common female malignant tumor types, which has a high mortality rate and a 5 year survival rate of $<50 \%$ (1-3). The poor prognosis of patients with ovarian cancer is largely attributed to the intrinsic molecular changes that occur, and certain oncogenes and tumor suppressors have been determined to serve key functions in ovarian cancer growth $(4,5)$. Therefore, a better understanding of the molecular mechanism that underlies ovarian cancer development and progression is urgently required for the development of novel therapeutic strategies.

The human genome encodes a large number of non-coding RNAs, including microRNAs (miRs) and long non-coding RNAs (lncRNAs) (6-10). miRs directly bind to the 3'-untranslated region (3'-UTR) of their target mRNAs, which leads to translation repression or mRNA degradation $(4,11,12)$. In addition to targeting numerous protein-coding genes, miRs can also target lncRNAs (13).

The lncRNA growth arrest-specific transcript 5 (GAS5), which is located at 1q25 and contains 630 nucleotides (6), has been reported to serve a tumor suppressive function in several common cancer types, including liver (13), breast (14), lung (15), renal (16) and colorectal cancer (17). In ovarian cancer, the downregulation of GAS5 promotes tumor cell proliferation, migration and invasion and indicates poor patient prognosis (18). Furthermore, Gao et al (19) reported that GAS5 could induce ovarian cancer cell apoptosis via the disruption of mitochondrial membrane potential and the promotion of pro-apoptotic protein expression, including Bcl-2-associated X, Bcl-2 homologous 
antagonist killer, cleaved-caspase 3 and cleaved-caspase 9. However, to the best of our knowledge, the underlying molecular mechanism through which GAS5 participates in ovarian cancer growth has not been previously studied.

miR-21 has been demonstrated to be upregulated in numerous cancer types, including ovarian cancer, and has been demonstrated to act as an oncogene (20-22). Targeting miR-21-3p could inhibit the proliferation and invasiveness of ovarian cancer cells (20). Recently, the targeting relationship between miR-21 and GAS5 has been reported in multiple common cancer types, including liver (13), lung (15), cervical (23) and breast cancer (14). However, to the best of our knowledge, the underlying mechanism by which GAS5 regulates miR-21 expression in ovarian cancer has not been previously reported. In addition, Sprouty homolog 2 (SPRY2), a member of the Sprouty family, has been demonstrated to serve a suppressive function in ovarian cancer. Patients with ovarian cancer whose tumors express SPRY2 at low levels have a significantly poorer prognosis compared with those who have tumors with high SPRY2 expression (24). However, the regulatory mechanism underlying SPRY2 expression in ovarian cancer remains unclear.

The aim of the current study was to investigate the underlying mechanism by which GAS5 regulates ovarian cancer cell proliferation, and the involvement of miR-21 and SPRY2 in this process.

\section{Materials and methods}

Clinical samples. Ovarian cancer tissues as well as adjacent normal tissues were collected from 53 patients with ovarian cancer at The First Affiliated Hospital of Xinxiang Medical University (Weihui, China) between June 2013 and April 2016. These 53 female patients were between 37 and 69 years old, with a mean age of 58.3 years old. These patients were assessed using FIGO staging (25). The clinical characteristics of these patients are summarized in Table I. None of the patients received radiation therapy or chemotherapy prior to surgical resection. Inclusion criteria were as follows: i) Primary surgical patient, ii) complete records regarding pre-operative chemotherapy and past medical history. Exclusion criteria were as follows: i) Non-primary surgical patient, ii) missing or incomplete records regarding pre-operative chemotherapy or past medical history, iii) previous history of any malignancy. Following resection, tissues were immediately stored in liquid nitrogen until use. The present study was approved by the Ethics Committee of The First Affiliated Hospital of Xinxiang Medical University (Weihui, China). Written informed consent was obtained from all patients.

Cell culture and transfection. Human ovarian cancer cell lines, OVCAR-3, SKOV3, 3AO, ES-2, HO-8910, A2780 and $\mathrm{COC} 1$, as well as the normal human ovarian epithelial cell line HOSEpiC, were purchased from the Type Culture Collection of the Chinese Academy of Sciences (Shanghai, China). All cells were cultured in Dulbecco's modified Eagle's medium (DMEM, Thermo Fisher Scientific, Inc., Waltham, MA, USA) supplemented with $10 \%$ fetal bovine serum (Thermo Fisher Scientific, Inc.) at $37^{\circ} \mathrm{C}$ with $5 \% \mathrm{CO}_{2}$ and were passaged every other day using $0.25 \%$ trypsin (Thermo Fisher Scientific, Inc.).
A2780 cells were transfected with 50 nM GAS5 expression plasmid (Yearthbio, Changsha, China), blank vector (Yearthbio), miR-21 inhibitor (anti-miR-21, cat. no. YB00238; Yearthbio) or negative control (NC) inhibitor (anti-NC, cat. no. YB00102; Yearthbio), or were co-transfected with either $50 \mathrm{nM}$ GAS5 expression plasmid and $50 \mathrm{nM}$ miR-21 mimic (cat. no. YB00237; Yearthbio), 50 nM GAS5 expression plasmid and $50 \mathrm{nM}$ scrambled miR mimic (miR-NC, Yearthbio), $50 \mathrm{nM} \mathrm{GAS5}$ expression plasmid and $50 \mathrm{nM}$ SPRY2 small interfering (si)RNA (cat. no. YB00588; Yearthbio) or $50 \mathrm{nM}$ GAS5 expression plasmid and $50 \mathrm{nM}$ NC siRNA (cat. no. YB00501; Yearthbio) using Lipofectamine 2000 (Thermo Fisher Scientific, Inc.), according to the manufacturer's protocol. Subsequent experiments were conducted at $48 \mathrm{~h}$ following transfection.

Reverse transcription-quantitative polymerase chain reaction $(R T-q P C R)$. Total RNA was extracted from tissues and cell lines using TRIzol reagent (Thermo Fisher Scientific, Inc.), according to the manufacturer's protocol. A total of $1 \mu \mathrm{g}$ RNA was converted into cDNA using a SuperScript ${ }^{\circledR}$ One-Step RT-PCR kit (Thermo Fisher Scientific, Inc.), according to the manufacturer's protocol. qPCR was performed to examine the expression levels of GAS5, miR-21 and SPRY2 mRNA using the Applied Biosystems ${ }^{\mathrm{TM}}$ PowerUp ${ }^{\mathrm{TM}}$ SYBR Green Master Mix kit (Thermo Fisher Scientific, Inc.) in an ABI 7500 fluorescence qPCR machine (Applied Biosystems; Thermo Fisher Scientific, Inc.), according to the manufacturer's protocol. U6 was used as the internal reference for GAS5 and miR-21, while GAPDH was used as the internal reference for SPRY2. The PCR reaction conditions were as follows: $95^{\circ} \mathrm{C}$ for $3 \mathrm{~min}$, followed by 40 cycles at $95^{\circ} \mathrm{C}$ for $15 \mathrm{sec}$ and $60^{\circ} \mathrm{C}$ for $30 \mathrm{sec}$. The relative expression levels were analyzed using the $2^{-\Delta \Delta C q}$ method (26). The primer sequences used are shown in Table II.

MTT assay. An MTT assay was performed to assess cell proliferation. A2780 cells (5,000 cells per well) were seeded in 96-well plates, and $100 \mu 1$ DMEM containing $0.5 \mathrm{~g} / 1$ MTT (Thermo Fisher Scientific, Inc.) was added into each well. Following incubation at $37^{\circ} \mathrm{C}$ for $12,24,48$ or $72 \mathrm{~h}$, the medium was removed and $50 \mu \mathrm{l}$ dimethyl sulfoxide (Thermo Fisher Scientific, Inc.) was added into each well. The cells were then incubated at $37^{\circ} \mathrm{C}$ for $10 \mathrm{~min}$. Cell proliferation was measured at a wavelength of $570 \mathrm{~nm}$ using the Varioskan LUX Multimode Microplate Reader (Thermo Fisher Scientific, Inc.).

Bioinformatics analysis. Bioinformatics analysis was performed to explore the targeting relationship among GAS5, miR-21 and SPRY2 using the miRecords resource and PicTar algorithms (c1.accurascience.com/miRecords/).

Luciferase reporter gene assay. Firefly luciferase reporter plasmids containing either wild-type (WT) miR-21 with GAS5 binding sequences, mutant-type (MT) miR-21 with no GAS5 binding sequences, WT SPRY2 3'-UTR with miR-21 binding sequences or MT SPRY2 3'-UTR without miR-21 binding sequences were purchased from Shanghai Genechem Co., Ltd. (Shanghai, China). To study the targeting relationship between GAS5 and miR-21, A2780 cells were co-transfected with a GAS5 plasmid or its control vector, and a WT or MT 
Table I. Association between growth arrest-specific transcript 5 expression and clinicopathological characteristics of patients with ovarian cancer.

\begin{tabular}{|c|c|c|c|c|}
\hline Variable & Cases $(n=53)$ & Low expression $(n=28)$ & High expression $(n=25)$ & P-value \\
\hline Age, years & & & & 0.578 \\
\hline$<55$ & 22 & 13 & 9 & \\
\hline$\geq 55$ & 31 & 15 & 16 & \\
\hline Differentiation & & & & 0.074 \\
\hline Well/moderate & 38 & 17 & 21 & \\
\hline Poor & 15 & 11 & 4 & \\
\hline FIGO stage & & & & $0.011^{\mathrm{a}}$ \\
\hline I-II & 32 & 12 & 20 & \\
\hline III-IV & 21 & 16 & 5 & \\
\hline
\end{tabular}

${ }^{a} \mathrm{P}<0.05$. FIGO, International Federation of Gynecology and Obstetrics.

Table II. Primers used in quantitative polymerase chain reaction.

\begin{tabular}{lll}
\hline Primer & \multicolumn{1}{c}{ Forward (5'-3') } & Reverse (5'-3') \\
\hline miR-21 & CAGTGCGTGTCGTGGAGT & ACGGGTAGCTTATCAGACTGA \\
U6 & CAAATTCGTGAAGCGTTCCATA & AGTGCAGGGTCCGAGGTATTC \\
GAS5 & CGACTCCTGTGAGGTATGGTG & ATCCTTCCTTGGGGACACAAC \\
SPRY2 & CCTACTGTCGTCCCAAGACCT & GGGGCTCGTGCAGAAGAAT \\
GAPDH & GGAGCGAGATCCCTCCAAAAT & GGCTGTTGTCATACTTCTCATGG
\end{tabular}

miR, microRNA; GAS5, growth arrest-specific transcript 5; SPRY2, Sprouty homolog 2.

miR-21 luciferase reporter plasmid using Lipofectamine 2000 (Thermo Fisher Scientific, Inc.), according to the manufacturer's protocol. To clarify the targeting relationship between miR-21 and SPRY2, A2780 cells were co-transfected with miR-21 mimic or miR-NC, and a WT or MT SPRY2 3'-UTR luciferase reporter plasmid using Lipofectamine 2000 (Thermo Fisher Scientific, Inc.), according to the manufacturer's protocol. Following transfection for $48 \mathrm{~h}$, luciferase activity was determined using the Dual-Luciferase Reporter Assay system (Promega Corporation, Madison, WI, USA). The firefly luciferase activities were normalized to Renilla luciferase activity.

Western blot analysis. A2780 cells were lysed in cold radioimmunoprecipitation assay buffer (Thermo Fisher Scientific, Inc.). Then, the protein concentration was determined using the BCA Protein Assay kit (Thermo Fisher Scientific, Inc.) according to the manufacturer's protocol. Protein (50 $\mu \mathrm{g} / \mathrm{well})$ was separated using $12 \%$ SDS-PAGE and was then transferred to a polyvinylidene fluoride (PVDF) membrane (Thermo Fisher Scientific, Inc.). The PVDF membrane was blocked in 5\% non-fat dried milk in phosphate-buffered saline (PBS; Thermo Fisher Scientific, Inc.) at $4^{\circ} \mathrm{C}$ overnight. Following washing in PBS with Tween-20 (PBST) for $10 \mathrm{~min}$ at room temperature, the PVDF membrane was incubated with a rabbit anti-human SPRY2 antibody (1:50; cat. no. ab50317; Abcam, Cambridge, MA, USA) or a rabbit anti-human GAPDH antibody (1:50; cat. no. ab9485; Abcam) for $3 \mathrm{~h}$ at room temperature. Following washing in PBST for $10 \mathrm{~min}$ at room temperature, the PVDF membrane was incubated with a horseradish peroxidase conjugated goat anti-rabbit secondary antibody (1:5,000; cat. no. ab6721, Abcam) for $1 \mathrm{~h}$ at room temperature. Following another wash in PBST for $10 \mathrm{~min}$ at room temperature, the immune complex on the PVDF membrane was detected using the Enhanced Chemiluminescence Western Blotting kit (Thermo Fisher Scientific, Inc.), according to the manufacturer's protocol. Protein expression was determined using Image-Pro Plus software 6.0 (Media Cybernetics, Inc., Rockville, MD, USA), and GAPDH was used as the internal reference.

Statistical analysis. Data are presented as the mean \pm standard deviation. SPSS 19.0 software (IBM Corp., Armonk, NY, USA) was used for statistical analysis. Data were analyzed using Student's t-test for two-group comparisons or ANOVA for comparisons of multiple groups followed by Turkey's post hoc test. A chi-square test was used to evaluate associations between GAS5 expression and clinicopathological characteristics. $\mathrm{P}<0.05$ was considered to indicate a statistically significant difference.

\section{Results}

Expression of GAS5, miR-21 and SPRY2 in ovarian cancer. The expression of GAS5, miR-21 and SPRY2 was examined in 

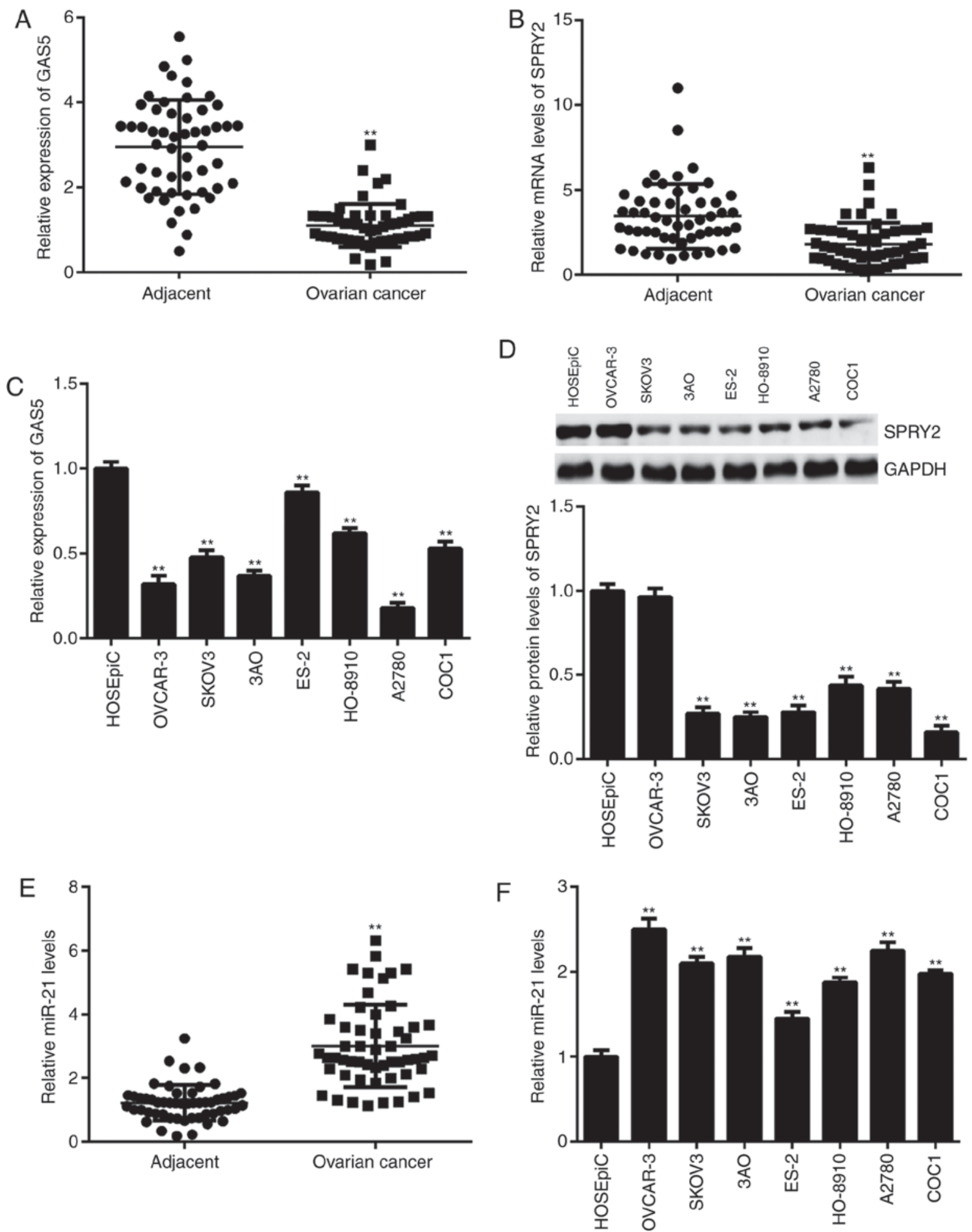

Figure 1. Expression of GAS5, SPRY2 and miR-21 in ovarian cancer tissues and cell lines. (A and B) qPCR was used to examine the expression levels of GAS5 and SPRY2 in ovarian cancer tissues compared with adjacent non-tumor tissues. " $\mathrm{P}<0.01$ vs. Adjacent. (C) qPCR and (D) western blot analysis were used to examine the expression levels of GAS5 and SPRY2 in ovarian cancer cell lines compared with normal ovarian epithelial cells. * P<0.01 vs. HOSEpiC. (E) qPCR was used to examine the miR-21 levels in ovarian cancer tissues compared with adjacent non-tumor tissues. ${ }^{* *} \mathrm{P}<0.01 \mathrm{vs}$. Adjacent. (F) qPCR was used to examine the miR-21 levels in ovarian cancer cell lines compared with normal ovarian epithelial cells. ${ }^{* *} \mathrm{P}<0.01$ vs. HOSEpiC. GAS5, growth arrest-specific transcript 5; SPRY2, Sprouty homolog 2; qPCR, quantitative polymerase chain reaction; miR, microRNA.

ovarian cancer tissues and cell lines. As shown in Fig. 1A and B, the expression of GAS5 and SPRY2 was significantly downregulated in ovarian cancer tissues compared with adjacent non-tumor tissues. Similar findings were also obtained in ovarian cancer cell lines except for OVCAR-3, when compared with normal ovarian epithelial cells (Fig. 1C and D). In addition, downregulation of GAS5 was significantly associated with advanced clinical stage in patients with ovarian cancer 
A WT miR-21 3'-...GUUGUAGUCAGACUAUUGGAU...-5'

GAS5 5'-...AGGCATTAGACAGA--AAGCTG...--3'

MT miR-21 3'-...GUUCATTAGACAGAAAAGCTG...-5'

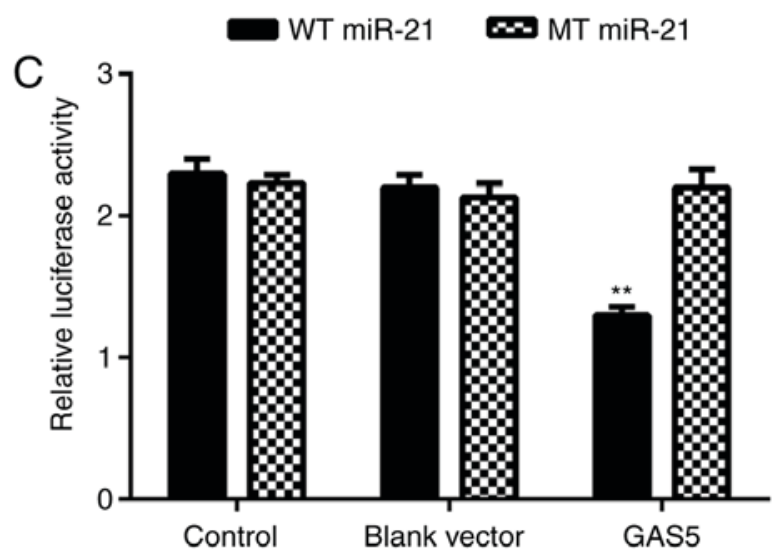

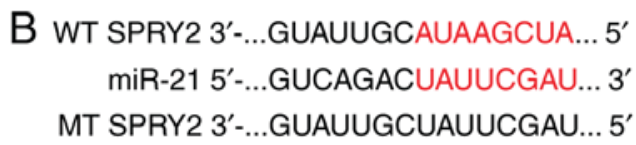

B WT SPRY2 $3^{\prime}$-...GUAUUGCAUAAGCUA... 5'

miR-21 5'-...GUCAGACUAUUCGAU... $3^{\prime}$

MT SPRY2 $3^{\prime}-\ldots$ GUAUUGCUAUUCGAU... $5^{\prime}$

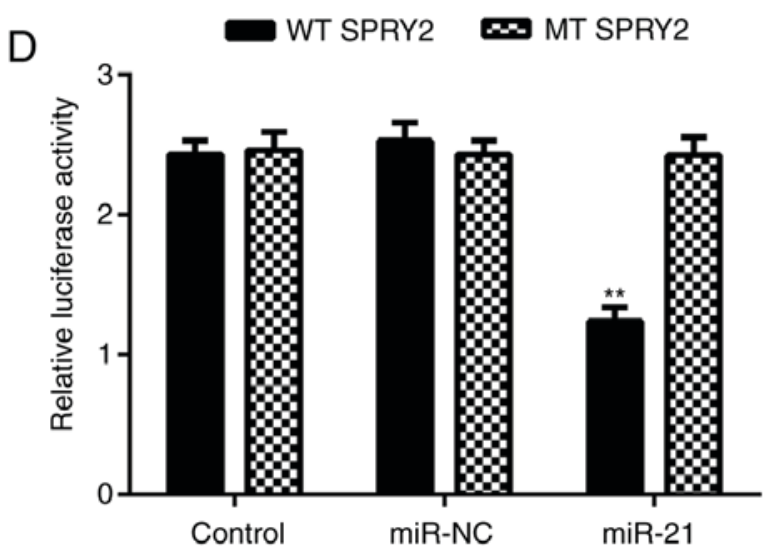

Figure 2. Luciferase reporter gene assay. (A) Bioinformatics analysis indicates that miR-21 contains GAS5 binding sites, and the WT and MT of miR-21 are shown. (B) Bioinformatics analysis indicates that SPRY2 3'-UTR contains miR-21 binding sites, and the WT and MT SPRY2 3'-UTR are shown. (C) A2780 cells co-transfected with GAS5 expression plasmid and WT or MT miR-21 reporter plasmid. (D) A2780 cells co-transfected with miR-21 mimic and WT or MT SPRY2 3'-UTR reporter plasmid. ${ }^{* *}$ P<0.01 vs. Control. GAS5, growth arrest-specific transcript 5; SPRY2, Sprouty homolog 2; WT, wild-type; MT, mutant-type; miR, microRNA; UTR, untranslated region.

(Table I). By contrast, the expression of miR-21 was significantly higher in ovarian cancer tissues and cell lines compared with adjacent non-tumor tissues and normal ovarian epithelial cells, respectively (Fig. 1E and F). In summary, these findings demonstrate that GAS5 and SPRY2 are downregulated, while miR-21 is upregulated, in ovarian cancer. As A2780 cells showed the lowest levels of GAS5, they were selected for use in subsequent experiments.

miR-21 is a target of GAS5 and SPRY2 is a target gene of miR-21 in A2780 cells. Bioinformatics analysis was performed to explore the targeting relationship among GAS5, miR-21 and SPRY2 using the miRecords resource and PicTar algorithms (c1.accurascience.com/miRecords/). The data indicated that miR-21 contained GAS5 binding sites and SPRY2 3'-UTR contained miR-21 binding sites (Fig. 2A and B). Therefore, miR-21 may be a target of GAS5, and SPRY2 may be a target gene of miR-21 in ovarian cancer cells. To clarify these predictions, a luciferase reporter assay was performed. The results indicated that ovarian cancer cells that were co-transfected with a GAS5 expression plasmid and a WT miR-21 reporter plasmid exhibited significantly reduced luciferase activity compared with the control group, but cells that were co-transfected with a GAS5 expression plasmid and an MT miR-21 reporter plasmid exhibited no effect on reporter luciferase activity (Fig. 2C). Therefore, miR-21 was demonstrated to be a target of GAS5 in ovarian cancer cells. Similarly, ovarian cancer cells that were co-transfected with an miR-21 mimic and a WT SPRY2 3'-UTR reporter plasmid exhibited a significant decrease in luciferase activity compared with the control group, but cells that were co-transfected with an miR-21 mimic and an MT SPRY2 reporter plasmid demonstrated no difference with respect to luciferase activity (Fig. 2D). Thus, SPRY2 was identified to be a target gene of miR-21 in ovarian cancer A2780 cells.
Overexpression of GAS5 inhibits A2780 cell proliferation and affects the expression of miR-21 and SPRY2. Based on the finding that GAS5 was significantly downregulated in ovarian cancer, A2780 cells were transfected with a GAS5 plasmid. Following transfection, the expression of GAS5 was significantly increased in the GAS5 group compared with the control group (Fig. 3A). The results from the MTT assay further indicated that, compared with the control group, GAS5 overexpression significantly decreased A2780 cell proliferation at $72 \mathrm{~h}$ (Fig. 3B). Furthermore, the effects of GAS5 on the expression of miR-21 and SPRY2 were studied. The data indicated that overexpression of GAS5 resulted in a significant decrease in miR-21 expression and a significant increase in SPRY2 expression in A2780 cells (Fig. 3C and D). Therefore, GAS5 could inhibit ovarian cancer cell proliferation and affect the expression of miR-21 and SPRY2.

Overexpression of miR-21 reduces SPRY2 expression and rescues GAS5-mediated A2780 cell proliferation. The effects of miR-21 downregulation on ovarian cancer cell proliferation and SPRY2 expression were studied. Since the expression of miR-21 was significantly upregulated in ovarian cancer tissues and cell lines, A2780 cells were transfected with an miR-21 inhibitor to knock down its expression. qPCR data indicated that miR-21 levels were significantly reduced in the anti-miR-21 group compared with the control group, but transfection with an NC inhibitor did not affect miR-21 levels (Fig. 4A). Furthermore, it was identified that the knockdown of miR-21 significantly inhibited A2780 cell proliferation at $72 \mathrm{~h}$, which was accompanied by significantly increased expression of SPRY2 (Fig. 4B and C).

To further confirm that miR-21 acts as the downstream effector of GAS5, A2780 cells were co-transfected with a GAS5 expression plasmid and a miR-21 mimic. Following transfection, the expression of miR-21 was significantly 

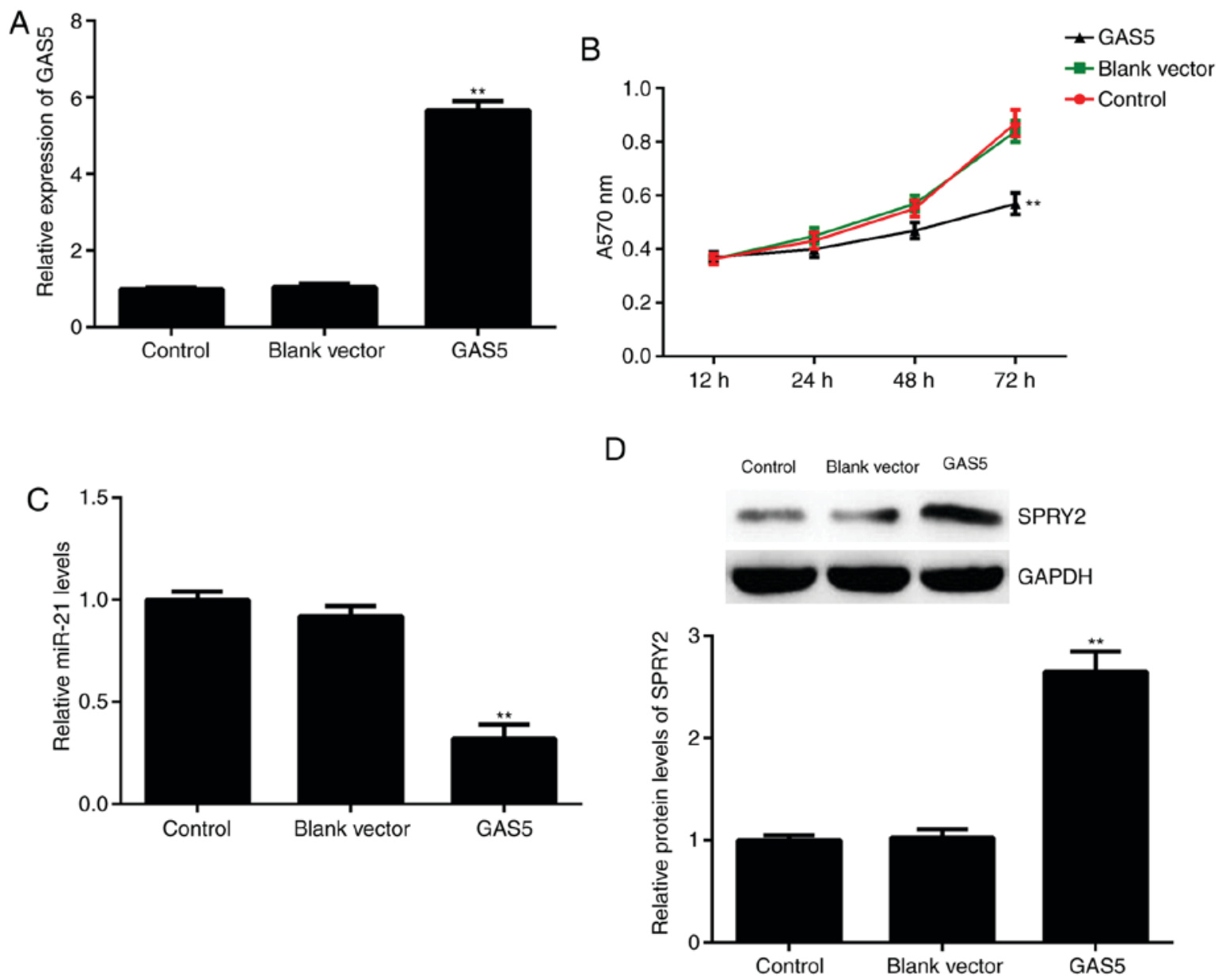

Figure 3. Effects of overexpression of GAS5. A2780 cells were transfected with GAS5 plasmid or blank vector. Non-transfected cells were used as the control group. Following transfection, (A) qPCR was used to examine GAS5 expression, (B) an MTT assay was used to determine cell proliferation, (C) qPCR was used to examine miR-21 levels and (D) western blot analysis was used to examine the protein levels of SPRY2. "* P $<0.01$ vs. Control. GAS5, growth arrest-specific transcript 5; SPRY2, Sprouty homolog 2; qPCR, quantitative polymerase chain reaction; miR, microRNA.

higher in the GAS5+miR-21 group compared with the GAS5+miR-NC group (Fig. 4D). Further investigation revealed that the proliferation of A2780 cells was significantly increased in the GAS5+miR-21 group at $72 \mathrm{~h}$ compared with the GAS5+miR-NC group, which suggests that miR-21 overexpression attenuated the suppressive effects of GAS5 on A2780 cell proliferation (Fig. 4E). In addition, the overexpression of miR-21 also attenuated the promoting effects of GAS5 on the expression of SPRY2 in A2780 cells (Fig. 4F).

Knockdown of SPRY2 rescues the inhibitory effects of GAS5 on A2780 cell proliferation. Finally, it was investigated whether SPRY2 was involved in GAS5-mediated ovarian cancer cell proliferation. A2780 cells were co-transfected with a GAS5 plasmid and SPRY2 siRNA. Following transfection, the mRNA and protein levels of SPRY2 were significantly downregulated in the GAS5+siSPRY2 group compared with the GAS5+siNC group (Fig. 5A and B). The results from the MTT assay indicated that the proliferation of A2780 cells was significantly increased in the GAS5+siSPRY2 group compared with the GAS5+siNC group at $72 \mathrm{~h}$ (Fig. 5C). These findings demonstrate that inhibition of SPRY2 rescues, at least partially, the suppressive effects of GAS5 overexpression on ovarian cancer cell proliferation.

\section{Discussion}

To the best of our knowledge, the underlying molecular mechanism through which lncRNA GAS5 regulates ovarian cancer growth has not been previously studied. In the present study, it was reported that GAS5 and SPRY2 were downregulated and that miR-21 was upregulated in ovarian cancer. Downregulation of GAS5 was significantly associated with advanced clinical stage of patients with ovarian cancer. miR-21 was identified to be a target gene of GAS5 and SPRY2 was identified to be a target gene of miR-21 in ovarian cancer A2780 cells. GAS5 overexpression significantly inhibited the proliferation of ovarian cancer cells, which was accompanied by the downregulation of miR-21 and the upregulation of SPRY2. Overexpression of miR-21 resulted in a significant decrease in A2780 cell proliferation, which was accompanied by reduced SPRY2 expression. Furthermore, miR-21 overexpression attenuated the suppressive effects of GAS5 on A2780 cell proliferation and rescued the promoting effects of GAS5 on SPRY2 expression. Furthermore, the knockdown of SPRY2 also rescued the suppressive effects of GAS5 on the proliferation of A2780 cells.

Recently, the association between GAS5 and miR-21 has been reported in multiple physiological and pathological 

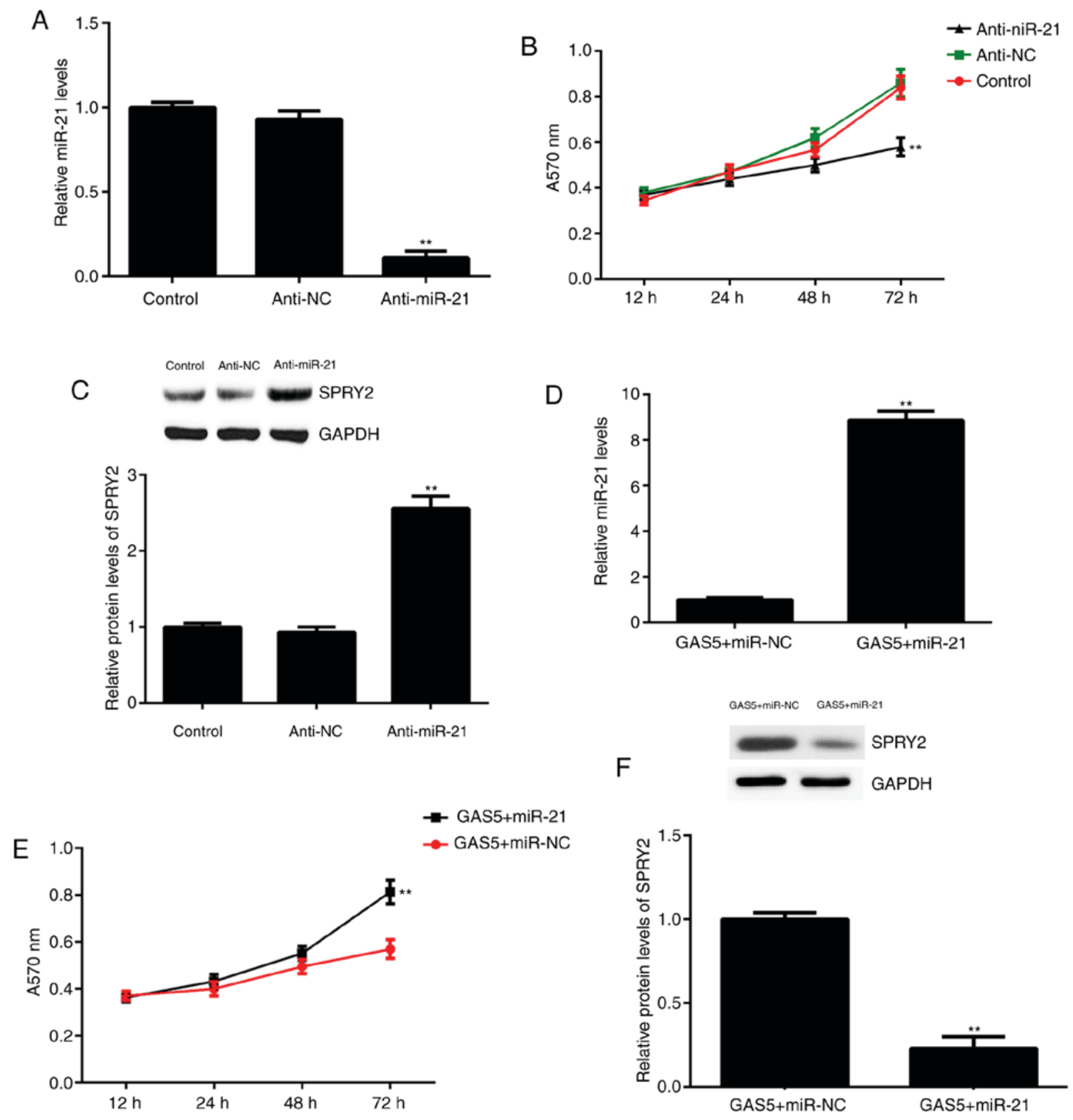

Figure 4. Effects of miR-21 inhibition and overexpression. A2780 cells were transfected with miR-21 inhibitor or NC inhibitor. Non-transfected cells were used as the control group. Following transfection, (A) qPCR was used to examine miR-21 levels, (B) an MTT assay was used to determine cell proliferation and (C) western blot analysis was used to examine the protein levels of SPRY2. " $\mathrm{P}<0.01$ vs. Control. Then, A2780 cells were co-transfected with GAS5 expression plasmid and miR-21 mimic or scramble miR (miR-NC). Following transfection, (D) qPCR was used to examine miR-21 levels, (E) an MTT assay was used to determine cell proliferation and $(\mathrm{F})$ western blot analysis was used to examine the protein levels of SPRY2. ${ }^{* *} \mathrm{P}<0.01$ vs. GAS5+miR-NC. GAS5, growth arrest-specific transcript 5; SPRY2, Sprouty homolog 2; qPCR, quantitative polymerase chain reaction; NC, negative control; miR, microRNA; anti-miR-21, miR-21 inhibitor.

processes (13-15). For instance, GAS5 inhibits cardiac fibroblast activation and fibrosis by targeting miR-21 through the phosphatase and tensin homolog (PTEN)/matrix metalloproteinase-2 signaling pathway (27). Furthermore, GAS5 contributes to the pathogenesis of osteoarthritis via the inhibition of miR-21 expression (28). Additionally, the tumor suppressive role of GAS5 has been reported in numerous cancer types (13). For instance, GAS5 suppresses cancer cell proliferation by acting as a molecular sponge for miR-21 and directly interacting with miR-21, which reverses the repression of PTEN, the endogenous target of miR-21 (14). Hu et al (13) reported that GAS5 suppresses the migration and invasion of hepatocellular carcinoma cells via the inhibition of miR-21. In addition, GAS5 and miR-21 are also involved in chemoresistance (15). Cao et al (15) demonstrated that inhibition of GAS5 reduces the chemosensitivity of non-small cell lung cancer cells to cisplatin through the upregulation of miR-21 and thus the downregulation of PTEN. In the present study, for the first time, the targeting relationship between miR-21 and GAS5 in ovarian cancer was reported. The present data indicated that GAS5 was significantly downregulated, while miR-21 was significantly upregulated, in ovarian cancer tissues. Luciferase 

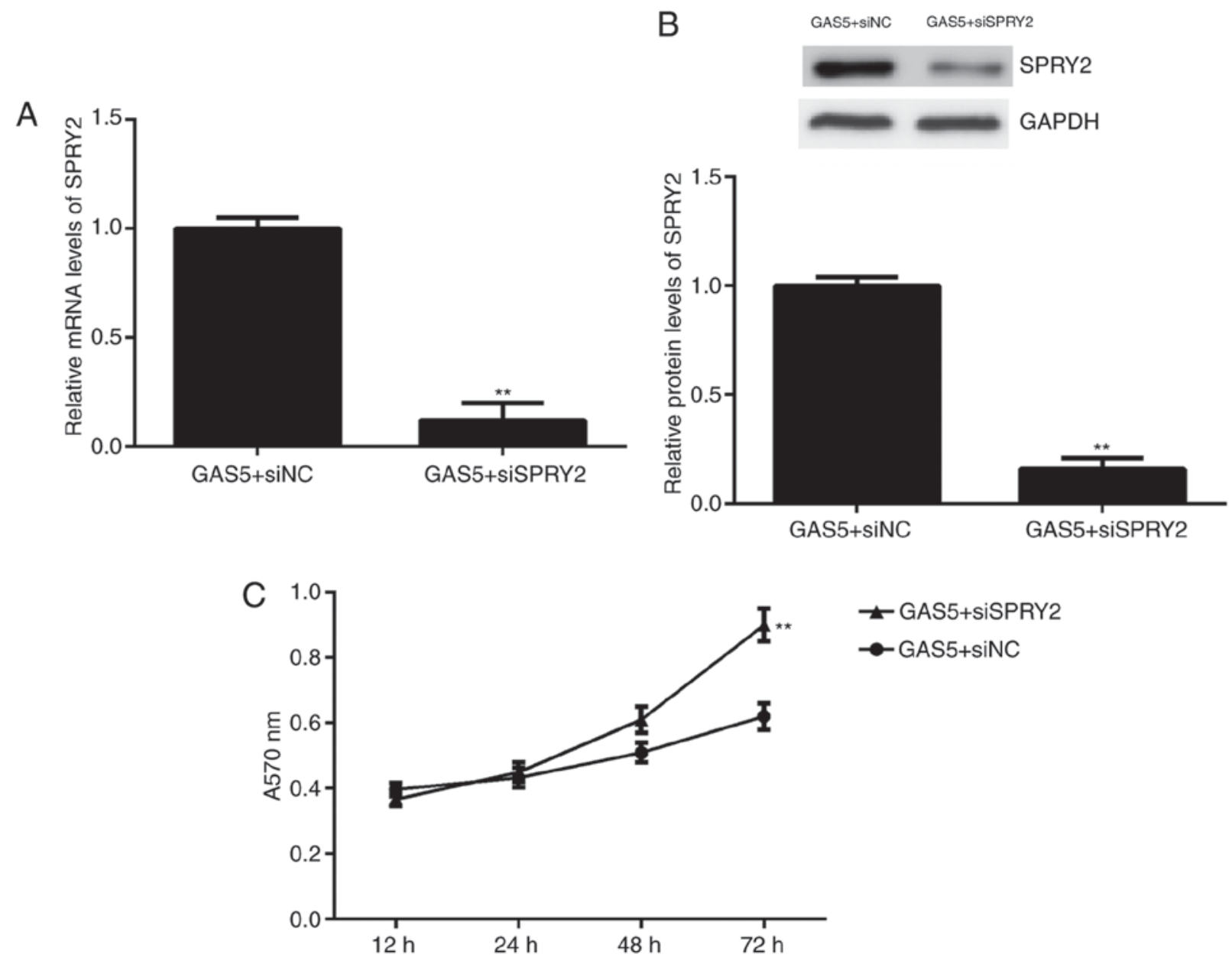

Figure 5. A2780 cells were co-transfected with GAS5 expression plasmid and SPRY2 siRNA or non-specific siRNA. Following transfection, (A) quantitative polymerase chain reaction and (B) western blot analysis were used to examine the mRNA and protein levels of SPRY2, respectively. (C) An MTT assay was used to determine cell proliferation. ${ }^{* *} \mathrm{P}<0.01$ vs. GAS5+siNC. siRNA, small interfering RNA; siNC, negative control siRNA; siSPRY2, SPRY2 siRNA; GAS5, growth arrest-specific transcript 5; SPRY2, Sprouty homolog 2.

reporter gene assay data confirmed that miR-21 was a direct target of GAS5 in A2780 cells and that the overexpression of GAS5 significantly reduced ovarian cancer cell proliferation by directly targeting miR-21.

Furthermore, it was identified in the current study that SPRY2 was also downregulated in ovarian cancer tissues, and luciferase reporter gene assay data confirmed that SPRY2 was a target gene of miR-21 in A2780 cells. SPRY2 is a member of the Sprouty family and inhibits the activity of receptor tyrosine kinase signaling; this protein is also required for its growth factor-stimulated translocation to membrane ruffles $(29,30)$. It has been widely demonstrated that SPRY2 acts as a tumor suppressor in some common human cancer types (31-33). For instance, SPRY2 is downregulated in renal cell carcinoma, and its low expression is associated with poor prognosis (34). Furthermore, SPRY2 may suppress the proliferation and invasiveness of renal cell carcinoma cells (34). Recently, Masoumi-Moghaddam et al (24) reported that SPRY2 is significantly downregulated in ovarian cancer, which is consistent with the present findings, and that patients whose tumors express SPRY2 at low levels have a significantly poorer prognosis compared with those who have tumors with high SPRY2 expression. In the present study, it was identified that SPRY2 is a direct target gene of miR-21 in A2780 cells. A targeting relationship between miR-21 and SPRY2 has also been identified in other cancer types (35,36). For instance, Kwak et al (35) reported that the downregulation of SPRY2 by miR-21 triggers malignancy in human gliomas. Huo et al (37) reported that disruption of pre-miR-21 sequences inhibits the proliferation, migration and invasiveness of ovarian cancer cells, which is accompanied by increased expression of SPRY2. In the present study, it was identified that GAS5 overexpression reduced miR-21 expression and increased the expression of its target gene, SPRY2. It was also demonstrated that the knockdown of SPRY2 expression rescued the inhibitory effects of GAS5 on A2780 cell proliferation. These findings suggest that SPRY2 is a downstream effector of GAS5/miR-21 signaling in A2780 cells.

To the best of our knowledge, this is the first study to report that GAS5 exerts inhibitory effects on ovarian cancer cell proliferation, at least in part through the downregulation of miR-21 expression and therefore the upregulation of SPRY2 expression. These findings suggest that the GAS5/miR-21/SPRY2 signaling pathway may be a potential therapeutic target in ovarian cancer. 


\section{Acknowledgements}

Not applicable.

\section{Funding}

No funding was received.

\section{Availability of data and materials}

All data generated or analyzed during this study are included in the published article.

\section{Authors' contributions}

SL collected the clinical tissues. NM, SL, QZ, HW and HQ performed the in vitro experiments. NM wrote the manuscript. SW designed the study and revised the manuscript.

\section{Ethics approval and consent to participate}

The present study was approved by the Ethics Committee of The First Affiliated Hospital of Xinxiang Medical University (Weihui, China). Written informed consent was obtained from all patients.

\section{Consent for publication}

Written informed consent for the publication of their data was obtained from all patients involved in the study.

\section{Competing interests}

The authors declare that they have no competing interests.

\section{References}

1. Siegel RL, Miller KD and Jemal A: Cancer statistics, 2015. CA Cancer J Clin 65: 5-29; 2015.

2. Torre LA, Bray F, Siegel RL, Ferlay J, Lortet-Tieulent J and Jemal A: Global cancer statistics, 2012. CA Cancer J Clin 65: 87-108, 2015.

3. Siegel R, Naishadham D and Jemal A: Cancer statistics, 2013. CA Cancer J Clin 63: 11-30, 2013.

4. Wang Y, Li J, Xu C and Zhang X: MicroRNA-139-5p inhibit cell proliferation and invasion by targeting RHO-associated coiled-coil containing protein kinase 2 in ovarian cancer. Oncol Res: Jun 14, 2017.

5. Hua F, Li CH, Chen XG and Liu XP: Long noncoding RNA CCAT2 knockdown suppresses tumorous progression by sponging miR-424 in epithelial ovarian vancer. Oncol Res 26 : 241-247, 2018.

6. Ebert MS and Sharp PA: Emerging roles for natural microRNA sponges. Curr Biol 20: R858-R861, 2010.

7. Li J, Zi Y, Wang W and Li Y: Long noncoding RNA MEG3 inhibits cell proliferation and metastasis in chronic myeloid leukemia via targeting miR-184. Oncol Res 26: 297-305, 2018.

8. Wang S, Hui Y, Li X and Jia Q: Silencing of lncRNA-CCDC26 restrains the growth and migration of glioma cells in vitro and in vivo via targeting miR-203. Oncol Res: Jun 9, 2017.

9. Zhang Y, Dai Q, Zeng F and Liu H: WITHDRAWN: MALAT1 promotes the proliferation and metastasis of osteosarcoma cells by activating the Rac1/JNK pathway via targeting miR-509. Oncol Res: May 26, 2017

10. Li Z, Guo J, Ma Y, Zhang L and Lin Z: Oncogenic role of MicroRNA-30b-5p in glioblastoma through targeting proline-rich transmembrane protein 2. Oncol Res 26: 219-230, 2018.
11. Yang M, Zhai X, Ge T, Yang C and Lou G: miR-181a-5p promotes proliferation and invasion, and inhibits apoptosis of cervical cancer cells via regulating inositol polyphosphate-5-phosphatase A (INPP5A). Oncol Res: Jun 23, 2017 (Epub ahead of print).

12. Zhou Y, Yang C, Wang K, Liu X and Liu Q: MicroRNA-33b inhibits the proliferation and migration of osteosarcoma cells via targeting hypoxia-inducible factor-1 $\alpha$. Oncol Res 25: 397-405, 2017.

13. Hu L, Ye H, Huang G, Luo F, Liu Y, Yang X, Shen J, Liu Q and Zhang J: Long noncoding RNA GAS5 suppresses the migration and invasion of hepatocellular carcinoma cells via miR-21. Tumour Biol 37: 2691-2702, 2016.

14. Li W, Zhai L, Wang H, Liu C, Zhang J, Chen W and Wei Q: Downregulation of LncRNA GAS5 causes trastuzumab resistance in breast cancer. Oncotarget 7: 27778-27786, 2016.

15. Cao L, Chen J, Ou B, Liu C, Zou Y and Chen Q: GAS5 knockdown reduces the chemo-sensitivity of non-small cell lung cancer (NSCLC) cell to cisplatin (DDP) through regulating miR-21/PTEN axis. Biomed Pharmacother 93: 570-579, 2017.

16. Qiao HP, Gao WS, Huo JX and Yang ZS: Long non-coding RNA GAS5 functions as a tumor suppressor in renal cell carcinoma. Asian Pac J Cancer Prev 14: 1077-1082, 2013.

17. Yang Y, Shen Z, Yan Y, Wang B, Zhang J, Shen C, Li T, Ye C, Gao Z, Peng G, et al: Long non-coding RNA GAS5 inhibits cell proliferation, induces G0/G1 arrest and apoptosis, and functions as a prognostic marker in colorectal cancer. Oncol Lett 13: 3151-3158, 2017.

18. Li J, Huang H, Li Y, Li L, Hou W and You Z: Decreased expression of long non-coding RNA GAS5 promotes cell proliferation, migration and invasion, and indicates a poor prognosis in ovarian cancer. Oncol Rep 36: 3241-3250, 2016.

19. Gao J, Liu M, Zou Y, Mao M, Shen T, Zhang C, Song S, Sun M, Zhang S, Wang B, et al: Long non-coding RNA growth arrest-specific transcript 5 is involved in ovarian cancer cell apoptosis through the mitochondria-mediated apoptosis pathway. Oncol Rep 34: 3212-3221, 2015.

20. Báez-Vega PM, Echevarría Vargas IM, Valiyeva F, Encarnación-Rosado J, Roman A, Flores J, Marcos-Martínez MJ and Vivas-Mejía PE: Targeting miR-21-3p inhibits proliferation and invasion of ovarian cancer cells. Oncotarget 7: 36321-36337, 2016.

21. Zhou L, Yang ZX, Song WJ, Li QJ, Yang F, Wang DS, Zhang N and Dou KF: MicroRNA-21 regulates the migration and invasion of a stem-like population in hepatocellular carcinoma. Int J Oncol 43: 661-669, 2013.

22. Yang X, Cheng Y, Li P, Tao J, Deng X, Zhang X, Gu M, Lu Q and Yin C: A lentiviral sponge for miRNA-21 diminishes aerobic glycolysis in bladder cancer T24 cells via the PTEN/PI3K/AKT/mTOR axis. Tumour Biol 36: 383-391, 2015.

23. Wen Q, Liu Y, Lyu H, Xu X, Wu Q, Liu N, Yin Q, Li J and Sheng X: Long noncoding RNA GAS5, which acts as a tumor suppressor via microRNA 21, regulates cisplatin resistance expression in cervical cancer. Int J Gynecol Cancer 27: 1096-1108, 2017.

24. Masoumi-Moghaddam S, Amini A, Wei AQ, Robertson G and Morris DL: Sprouty 2 protein, but not Sprouty 4, is an independent prognostic biomarker for human epithelial ovarian cancer. Int J Cancer 137: 560-570, 2015.

25. Javadi S, Ganeshan DM, Qayyum A, Iyer RB and Bhosale P: Ovarian cancer, the revised FIGO staging system, and the role of imaging. AJR Am J Roentgenol 206: 1351-1360, 2016.

26. Livak KJ and Schmittgen TD: Analysis of relative gene expression data using real-time quantitative PCR and the 2(-Delta Delta $\mathrm{C}(\mathrm{T})$ ) method. Methods 25: 402-408, 2001.

27. Tao H, Zhang JG, Qin RH, Dai C, Shi P, Yang JJ, Deng ZY and Shi KH: LncRNA GAS5 controls cardiac fibroblast activation and fibrosis by targeting miR-21 via PTEN/MMP-2 signaling pathway. Toxicology 386: 11-18, 2017.

28. Song J, Ahn C, Chun CH and Jin EJ: A long non-coding RNA, GAS5, plays a critical role in the regulation of miR-21 during osteoarthritis. J Orthop Res 32: 1628-1635, 2014.

29. Lim J, Wong ES, Ong SH, Yusoff P, Low BC and Guy GR: Sprouty proteins are targeted to membrane ruffles upon growth factor receptor tyrosine kinase activation. Identification of a novel translocation domain. J Biol Chem 275: 32837-32845, 2000.

30. Lim J, Yusoff P, Wong ES, Chandramouli S, Lao DH, Fong CW and Guy GR: The cysteine-rich sprouty translocation domain targets mitogen-activated protein kinase inhibitory proteins to phosphatidylinositol 4,5-bisphosphate in plasma membranes. Mol Cell Biol 22: 7953-7966, 2002. 
31. Liu C, Liang S, Xiao S, Lin Q, Chen X, Wu Y and Fu J: MicroRNA-27b inhibits Spry2 expression and promotes cell invasion in glioma U251 cells. Oncol Lett 9: 1393-1397, 2015.

32. Chandramouli S, Yu CY, Yusoff P, Lao DH, Leong HF, Mizuno K and Guy GR: Tesk1 interacts with Spry2 to abrogate its inhibition of ERK phosphorylation downstream of receptor tyrosine kinase signaling. J Biol Chem 283: 1679-1691, 2008.

33. Wu G, Qin XQ, Guo JJ, Li TY and Chen JH: AKT/ERK activation is associated with gastric cancer cell resistance to paclitaxel. Int J Clin Exp Pathol 7: 1449-1458, 2014.

34. Li P, Tao L, Yang J, Cai H, Ju X, Li J, Shao P, Cao Q, Qin C, Meng $X$ and Yin C: Sprouty2 is associated with prognosis and suppresses cell proliferation and invasion in renal cell carcinoma. Urology 82: 253.e1-e7, 2013.

35. Kwak HJ, Kim YJ, Chun KR, Woo YM, Park SJ, Jeong JA, Jo SH, Kim TH, Min HS, Chae JS, et al: Downregulation of Spry2 by miR-21 triggers malignancy in human gliomas. Oncogene 30: 2433-2442, 2011.
36. Feng YH, Wu CL, Shiau AL, Lee JC, Chang JG, Lu PJ, Tung CL, Feng LY, Huang WT and Tsao CJ: MicroRNA-21-mediated regulation of Sprouty 2 protein expression enhances the cytotoxic effect of 5-fluorouracil and metformin in colon cancer cells. Int J Mol Med 29: 920-926, 2012.

37. Huo W, Zhao G, Yin J, Ouyang X, Wang Y, Yang C, Wang B, Dong P, Wang Z, Watari H, et al: Lentiviral CRISPR/Cas9 vector mediated miR-21 gene editing inhibits the epithelial to mesenchymal transition in ovarian cancer cells. J Cancer 8: 57-64, 2017.

(i) () $($ This work is licensed under a Creative Commons Attribution-NonCommercial-NoDerivatives 4.0 International (CC BY-NC-ND 4.0) License. 